\title{
CEF1/CDC5 alleles modulate transitions between catalytic conformations of the spliceosome
}

\author{
CHARLES C. QUERY ${ }^{1,3}$ and MARIA M. KONARSKA ${ }^{2,3}$ \\ ${ }^{1}$ Department of Cell Biology, Albert Einstein College of Medicine, Bronx, New York 10461, USA \\ ${ }^{2}$ The Rockefeller University, New York, New York 10065, USA
}

\begin{abstract}
Conformational change within the spliceosome is required between the first and second catalytic steps of pre-mRNA splicing. A prior genetic screen for suppressors of an intron mutant that stalls between the two steps yielded both prp8 and non-prp8 alleles that suppressed second-step splicing defects. We have now identified the strongest non-prp8 suppressors as alleles of the NTC (Prp19 complex) component, CEF1. These cef1 alleles generally suppress second-step defects caused by a variety of intron mutations, mutations in U6 snRNA, or deletion of the second-step protein factor Prp17, and they can activate alternative 3' splice sites. Genetic and functional interactions between cef1 and prp8 alleles suggest that they modulate the same event(s) in the first-to-second-step transition, most likely by stabilization of the second-step spliceosome; in contrast, alleles of U6 snRNA that also alter this transition modulate a distinct event, most likely by stabilization of the first-step spliceosome. These results implicate a myb-like domain of Cef1/CDC5 in interactions that modulate conformational states of the spliceosome and suggest that alteration of these events affects splice site use, resulting in alternative splicing-like patterns in yeast.
\end{abstract}

Keywords: Cef1; NTC; Prp8; U6 snRNA; Prp22; intron mutations; fidelity

\section{INTRODUCTION}

Removal of introns from pre-mRNA is catalyzed by the spliceosome, a large, multicomponent complex whose assembly and function require multiple conformational transitions (for reviews, see Smith et al. 2008; Wahl et al. 2009). Splicing catalysis proceeds through two consecutive transesterifications involving three sites of the intron: in the first reaction, the branch site nucleophilically attacks the 5' splice site (SS), producing a lariat intermediate and cleaved $5^{\prime}$ exon, and, in the second reaction, the $5^{\prime}$ exon attacks the 3'SS yielding spliced mRNA and lariat intron products.

The phenotypes of many spliceosomal mutants can be explained by a two-state model of the catalytic spliceosome in which conformations of the complex supporting the two catalytic steps are in competition, with modulation of the relative stabilities of the first- and second-step conformations resulting in improvement of one of the catalytic steps

${ }^{3}$ Corresponding authors.

E-mail charles.query@einstein.yu.edu.

E-mail konarsk@rockefeller.edu.

Article published online ahead of print. Article and publication date are at http://www.rnajournal.org/cgi/doi/10.1261/rna.029421.111. to the detriment of the other. This model is supported by an analysis of two classes of spliceosomal alleles residing in PRP8, U6 snRNA, PRP16, and ISY1: "first-step alleles," which suppress first-step defects due to intron mutations while exacerbating second-step defects; and "second-step alleles," all of which similarly suppress a large number of intron mutations at 5'SS, branch site, and 3'SS by improving the second step while also reducing the efficiency of the first step (Query and Konarska 2004; Villa and Guthrie 2005; Liu et al. 2007).

Prp8, the largest and most highly conserved protein component of the spliceosome, can harbor mutations that fall within either of these two classes (Query and Konarska 2004; Liu et al. 2007) in addition to other classes of mutations (for review, see Grainger and Beggs 2005). Similarly, mutations of position U57 of U6 snRNA, originally identified as suppressors of branch site mutations (McPheeters 1996), also represent both general classes of suppressor alleles-in this case, U6-U57C improves the first step and inhibits the second, whereas U6-U57A improves the second step and inhibits the first, for a wide variety of intron mutations (McPheeters 1996; Query and Konarska 2004; Liu et al. 2007). Prp16, a member of the DExH/D family of RNA-dependent ATPase/helicases, facilitates the first-to-second-step transition (Schwer and 
Guthrie 1991); mutant alleles of prp16 are thought to improve the first step of splicing for mutant introns limiting for this step by slowing the exit from the first-step conformation of the spliceosome, which enhances first-step catalysis (for review, see Konarska and Query 2005; Koodathingal et al. 2010). In addition, the Prp22 ATPase/helicase facilitates exit from the second step and, thus, mRNA release (Schwer and Gross 1998); mutant alleles of prp22 improve secondstep catalysis for introns limiting for this step by slowing the exit from the second-step conformation (Mayas et al. 2006).

A previous genetic screen in Saccharomyces cerevisiae for suppressors of a branch site A-to-G mutation yielded a number of strains that improved the second step of splicing, some of which were identified as alleles of prp8 (Query and Konarska 2004). The strongest suppressor strains obtained in this screen, however, were not attributable to mutations in PRP8. Based on predictions of the two-state model, we hypothesized that these suppressors, which alone exhibit no growth defects, would exacerbate growth defects due to ATPase-defective alleles of prp22. We have confirmed this synthetic growth defect and used it to identify the remaining, strongest suppressors from this screen as alleles of CEF1/CDC5. Cef1 was originally identified as CDC5 in a screen for cell cycle mutants in Schizosaccharomyces pombe (Nurse et al. 1976) and was later shown to be a component of the NTC (Prp19 complex) that associates with the spliceosome to form the active complex (Tsai et al. 1999; for review, see Wahl et al. 2009). Cefl contains myblike domains that are often involved in double-stranded nucleic acid interactions-e.g., in c-myb, Rap1, or TRF1 and -2 proteins (Ohi et al. 1994). Mutations in Cef1 that improve the second step are restricted to two amino acid positions in the amino-terminal myblike domain, an observation we confirmed by saturation mutagenesis of the two Cefl myb-like domains.

These results implicate the $\mathrm{N}$-terminal myb-like domain of Cef1/CDC5 in interactions that modulate conformational states of the spliceosome. Thus, alleles of two spliceosomal proteins, cef 1 and $\operatorname{prp} 8$, can similarly alter both the first-to-second-step transition and release from the second-step conformation, globally supporting the previously proposed two-state model.

\section{RESULTS}

\section{Identification of $C E F 1 / C D C 5$ alleles as suppressors of the BS-G second-step defect}

Branch site A-to-G (BS-G) intron mutation permits efficient progression through the first step of splicing but results in a strong block to the second step (Fig. 1A), as revealed by primer extension of the RNA isolated from wild-type yeast cells. In addition, whereas the efficient splicing of the wild-type ACT1-CUP1 reporter gene (Lesser and Guthrie 1993) yields high levels of CUP1 mRNA, allowing cells to grow in the presence of copper, wild-type cells carrying the BS-G ACT1-CUP1 reporter are highly

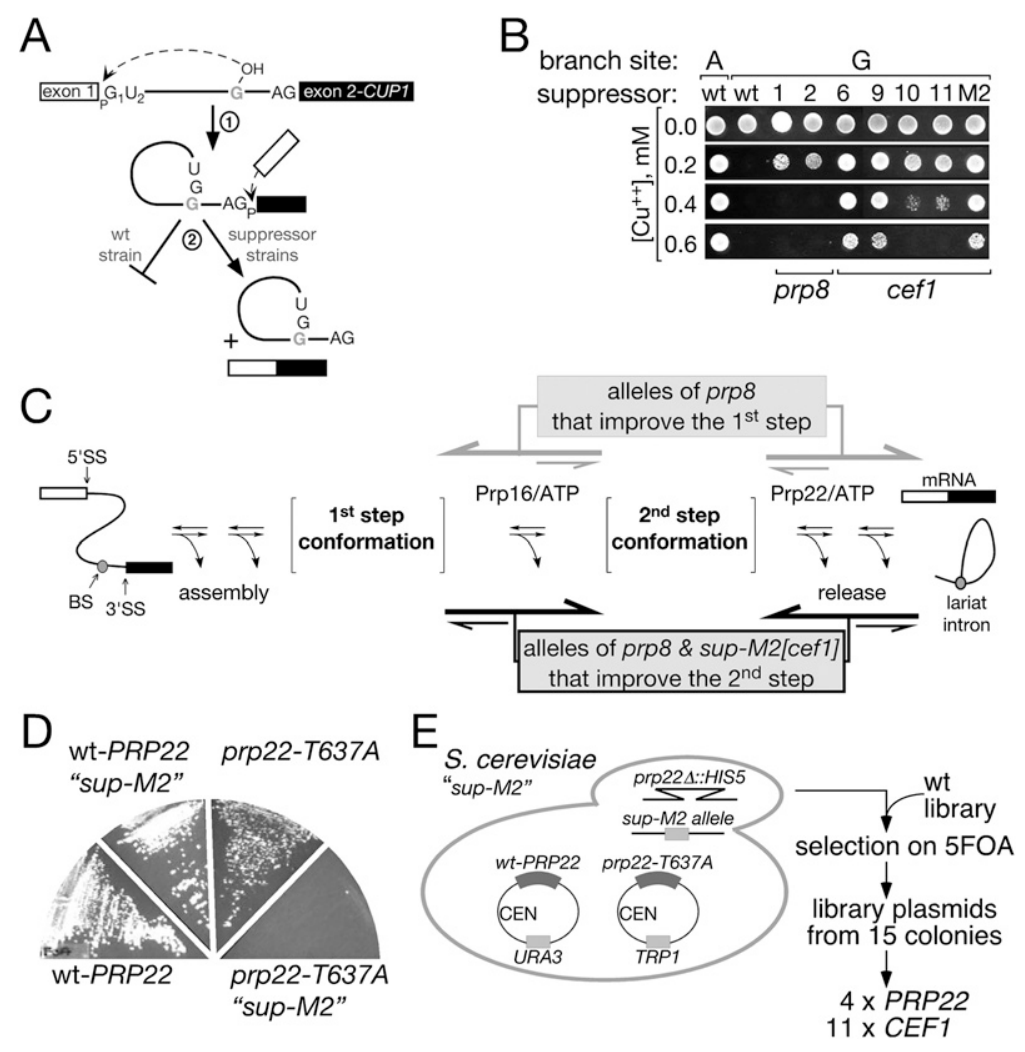

FIGURE 1. Synthetic lethality of suppressors of second-step splicing defects with prp22 alleles allows for their identification. (A) Schematic of splicing chemistry for BS-G mutant ACT1-CUP1 reporter in vivo. In wild-type cells, BS-G lariat intermediates stall prior to the second step of splicing and accumulate; in the isolated suppressor strains, BS-G lariat intermediates proceed through the second step, forming excised lariat and mRNA. (B) Copper growth phenotype of the BS-G mutant in the previously isolated BS suppressors. Suppressor strains 1 and 2 (containing prp8-161 and -162 alleles) (Query and Konarska 2004) and strains 6, 9, 10, 11, and M2 (containing alleles of $c e f 1$, reported here) support growth on $0.2 \mathrm{mM}$ or higher copper, whereas wild-type cells do not. $(C)$ Schematic representation of splicing pathway and assignment of mutant alleles of prp8, prp16, prp22 and U6 snRNA that modulate spliceosomal transitions. (SS) splice site, (BS) branch site. The non-prp8 suppressor alleles, which are thought to improve the second step by a relative stabilization of the second-step conformation, were predicted to exacerbate defects in the DEAH-ATPase Prp22p. (D) The non-prp8 suppressor "M2" is synthetically lethal with $\operatorname{prp} 22$ mutants at $30^{\circ} \mathrm{C}$. (E) Schematic of screen to identify the isolated suppressors, using rescue of the synthetic growth defect of the suppressor mutations in combination with helicase-defective prp22 alleles. Genomic library plasmids that rescued this growth defect carried CEF1 or wild-type PRP22. 
sensitive to copper addition to the growth medium (Fig. 1B). We isolated nine strains that suppressed the BS-G defect, strongly improving splicing of the reporter and thereby growth on copper in comparison to the wild-type strain (Fig. 1B); in two of these strains, we previously identified mutated alleles of $\operatorname{prp} 8$, which we characterized as general suppressors of second-step defects caused by intron mutations (Query and Konarska 2004).

Our model of competition between spliceosomal conformations predicts that suppressors of second-step defects would exacerbate defects in the subsequent conformational transition facilitated by Prp22 (Fig. 1C). We, therefore, hypothesized that other suppressors could be identified by the ability of an additional wild-type copy of the suppressor gene to rescue a negative growth phenotype conferred by the combination of the suppressor mutation and a mutant prp22 allele. Indeed, prp8 alleles that suppress second-step defects exacerbate growth defects due to mutations in the SAT motif of Prp22; in contrast, prp8 alleles that suppress first-step defects suppress prp22 defects (Schneider et al. 2004; Liu et al. 2007). This suggested that the non-prp8 suppressors of BS-G might also exacerbate prp22 defects. We, therefore, disrupted the PRP22 locus in the strongest suppressor strain, "M2," bearing PRP22 on a plasmid, and tested for synthetic phenotypes with various prp22 alleles. Indeed, whereas either the $M 2$ mutant or the cold-sensitive prp22-T637A mutant alone supported growth at $30^{\circ} \mathrm{C}$, yeast harboring the combination of these mutations grew slowly at $37^{\circ} \mathrm{C}$ and failed to grow at $30^{\circ} \mathrm{C}$ (Fig. $1 \mathrm{D}$ and data not shown); similar results were observed with prp22S635A (data not shown). This cold-sensitive phenotype was used to screen a genomic library for wild-type genes that would restore viability to this strain at $30^{\circ} \mathrm{C}$ (Fig. $1 \mathrm{E}$ ). Of 15 library plasmids that rescued the growth defect, four plasmids contained PRP22, as expected, and eleven plasmids contained CEF1, which tentatively identified the M2 suppressor as a cef1 allele. Sequencing of the cef1 alleles in five of the suppressor strains identified point mutations, and their ability to confer the suppressor phenotype was confirmed using plasmid-borne cef1 alleles (see Figs. 2-7, below). The cef1 suppressor mutations V36R and S48R were both isolated multiple times in our original screen (strains 6, 9, and M2, and 10 and 11, respectively, in Fig. 1B) and are located in the N-terminal myb-domain (see Fig. 4A, below).

\section{cef1 alleles are general suppressors of second-step defects}

To test whether the cefl mutants act as general suppressors of splicing defects, we first analyzed their effect on a variety of intron mutations at the $5^{\prime} \mathrm{SS}$, BS, and $3^{\prime} \mathrm{SS}$; splicing defects due to each of the mutations indicated (Fig. 2A) were suppressed, and a representative result for each site is shown (Fig. 2B). Strong improvement in the second step of
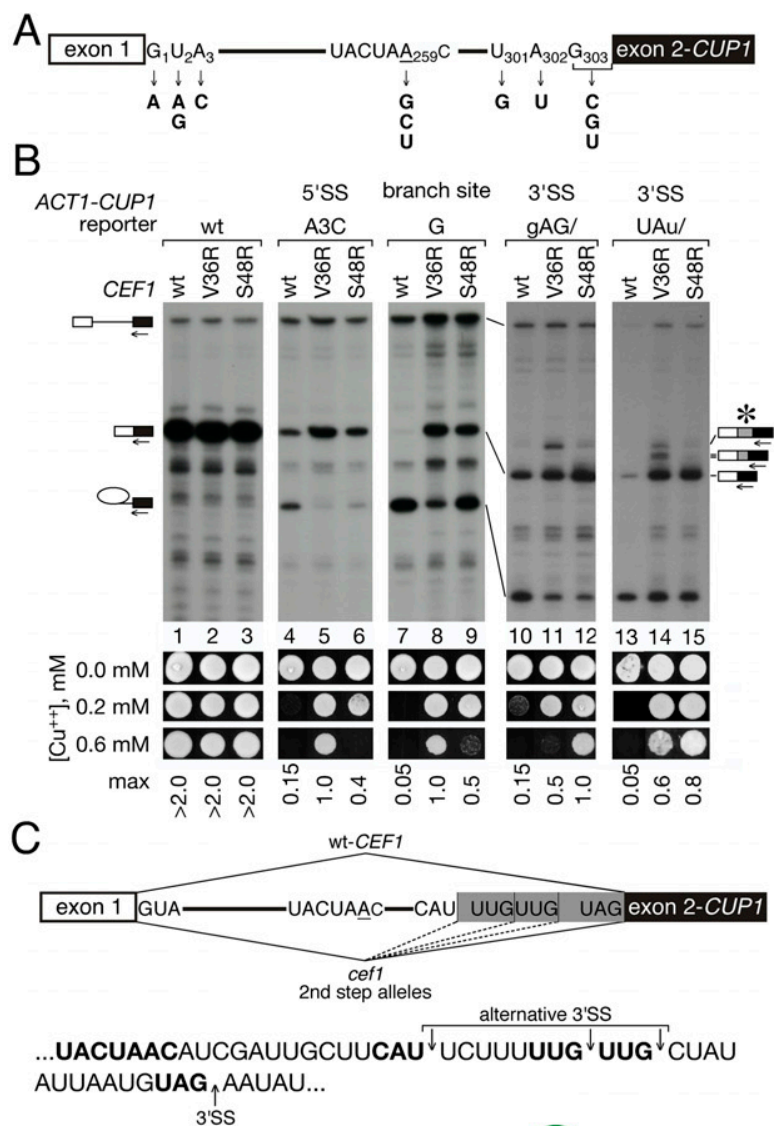

D

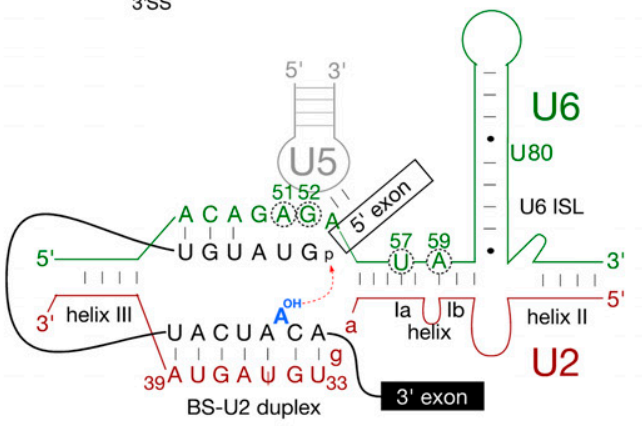

$E$

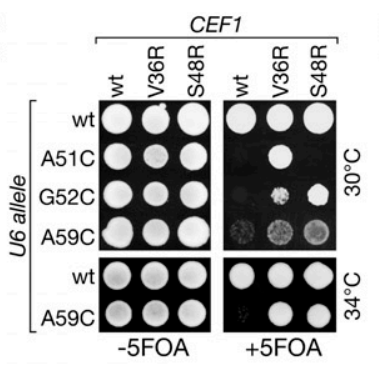

G
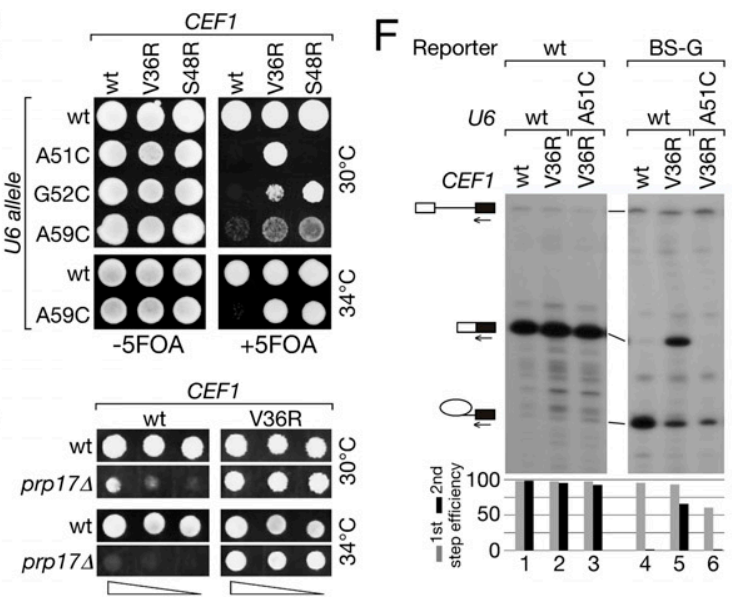

FIGURE 2. (Legend on next page) 
splicing was observed for multiple 5'SS mutations, BS-C, -G, and $-\mathrm{U}$ mutations, and all tested $3^{\prime} \mathrm{SS}$ mutations at positions $-3,-2$, and -1 , as indicated by increased levels of spliced mRNA, decreased levels of lariat intermediates, and improved growth on copper (Fig. 2B and data not shown). This strong second-step improvement was observed with either the V36R or S48R mutants. Concomitant with this, there was a reduced efficiency of the first step, most clearly visualized by a BS-C intron reporter that is inefficient for both steps of splicing (see Fig. 6B, lanes 1-3, or Fig. 7B, lanes 1-3, below).

To compare the strength of the effects on splicing exhibited by cef1 alleles to those of other suppressors of second-step defects, we analyzed the BS-G, 5'SS A3C, and 3'SS gAG/ reporters in strains carrying alleles of prp8, U6, and prp22 that have previously been shown to suppress second-step defects (McPheeters, 1996; Collins and Guthrie 1999; Siatecka et al. 1999; Query and Konarska 2004; Mayas et al. 2006; Liu et al. 2007). Among these suppressors, cef1 alleles exhibited by far the strongest improvement of the second step of splicing for the BS-G reporter, and improvement comparable to that of the strongest $\operatorname{prp} 8$ alleles was observed for the 5'SS A3C and 3'SS gAG/ reporters (Fig. 3). Although the relative potency of suppression effects of the cef1 alleles appears to vary among different reporters-for example, cefl-V36R improved BS-G and A3C reporters more than did cef1-S48R, and the opposite was the case for the $3^{\prime} \mathrm{SS}$ gAG/ reporter-this difference is likely due to activation of cryptic (and out of frame) 3 'SS by the cef1-V36R allele, discussed more below.

FIGURE 2. cef1 alleles are general suppressors of second-step splicing defects. (A) Schematic of ACT1-CUP1 pre-mRNA, indicating intron mutations at $5^{\prime}$ SS, BS, and $3^{\prime}$ SS used in $B$ and other mutations suppressed by cef1-V36R and -S48R. (B) cef1 alleles suppress multiple intron mutations. Upper, primer extension analysis of RNA from cells containing wild-type CEF1, cef1-V36R, or cef1-S48R and ACT1-CUP1 reporters as indicated. Primer complementary to the $3^{\prime}$ exon was used to monitor levels of pre-mRNA, mRNA, and lariat intermediate (indicated by icons on the left; icons on the right indicate use of alternative $3^{\prime}$ splice sites that are activated by cefl-V36R or $-48 R$ alleles). Lower, copper growth phenotypes of strains carrying the reporters used above; representative copper concentrations are shown, along with the highest concentration allowing for growth. (C) Sequence of the 3'SS region of the ACT1-CUP1 pre-mRNA, indicating the wild-type $3^{\prime} S S$ and the additional weak $3^{\prime}$ splice sites that are used in the presence of $c e f 1$ alleles. $(D)$ Schematic of RNA:RNA interactions in the spliceosome core, indicating nucleotides in U6 snRNA whose mutation inhibits the second step of splicing, used in panels $E$ and $F$, and in Figure 6. Pre-mRNA is shown in black, U2 snRNA in red, U5 snRNA in gray, and U6 snRNA in green; numbering corresponds to $S$. cerevisiae snRNAs. (E) cef1 alleles suppress the lethal growth defects of U6-A51C, -G52C, and -A59C. (F) U6-A51C inhibits the second step of splicing in vivo. Primer extension analysis (as in Fig. 2B) of RNA from cells containing U6-A51C allele and the BS-C reporter. First-step efficiency was calculated as products of the first step/total RNA. Second-step efficiency was calculated as products of the second step/total products from the first step. $(G)$ The cef1-V36R allele suppresses the growth defect of prp 17 deletion at $30^{\circ} \mathrm{C}$ and rescues viability at $34^{\circ} \mathrm{C}$; a series of $1: 5$ dilutions is shown.

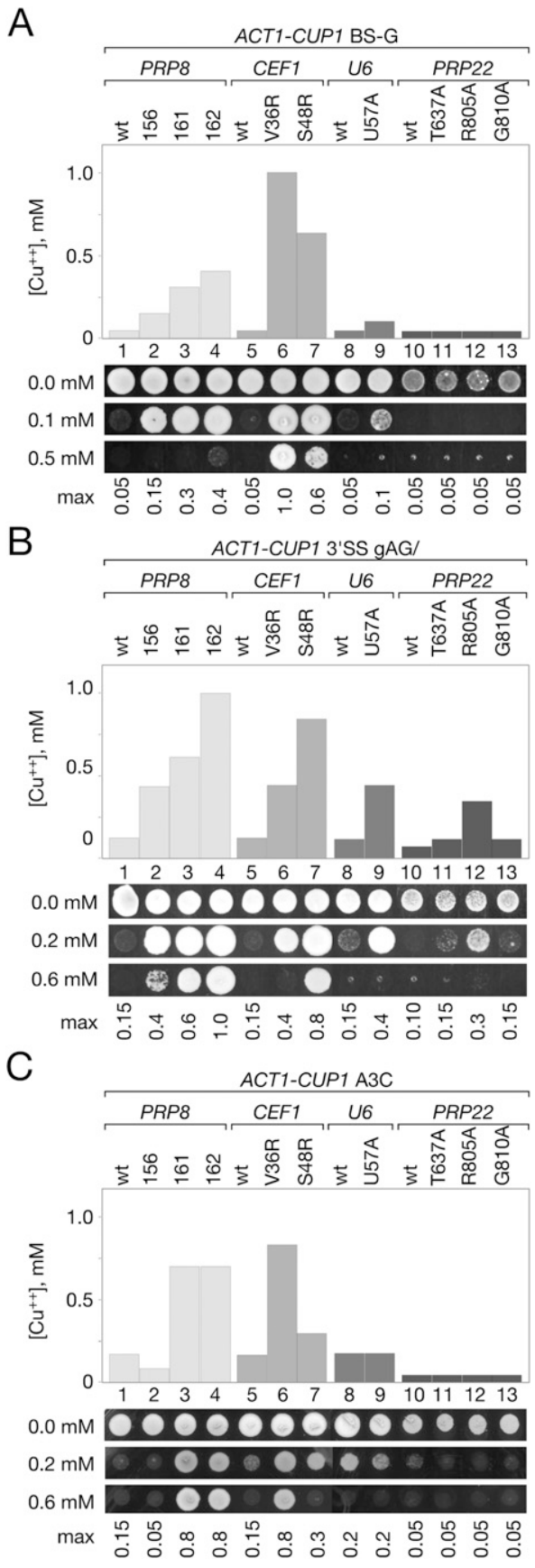

FIGURE 3. Comparison of the strength of second-step improvement by cef1 alleles to that of alleles of prp8, U6 snRNA, and prp22. $(A, B, C)$ Graphs (upper) summarizing the copper growth (lower) of strains carrying the indicated pairs of reporters and second-step alleles.

As a number of mutations in U6 snRNA that are known to inhibit the second step in vitro (Fabrizio and Abelson 1990; Madhani et al. 1990; Madhani and Guthrie 1992; Hilliker and Staley 2004) are unable to support cell growth (McPheeters 1996 and references therein), general suppressors of second-step defects might be expected to rescue lethality associated with these U6 mutants. To test this possibility, we combined several inviable U6 mutants with 
cef1-V36R and $-S 48 R$ alleles. Indeed, the cef1-V36R allele restored viability of a strain carrying U6-A51C alleles, and growth defects due to U6-G52C and -A59C alleles also were suppressed by each of the cef1 alleles (Fig. 2E).

The complementation of the U6-A51C mutant by the cef1-V36R allele allowed a not-previously-possible analysis of its effect on splicing in vivo. In the presence of cef1$V 36 R$, splicing of the wild-type reporter intron was not affected (Fig. 2F, lanes 1-3). However, for BS-G, whose second step is strongly improved by cef 1 alleles, the second step is strongly inhibited by U6-A51C, and the efficiency of the first step is also reduced (Fig. 2F, cf. lanes 6 and 5). Thus, the dominant effect observed for U6-A51C in vivo is an inhibition of the second step, and suppression of this defect by the cef 1 allele restored viability.

Prp17, also known as CDC40, contributes to the second step and, like several other second-step factors (Slu7, Prp22, Prp18), facilitates the splicing of introns containing long BS-3'SS distances (Sapra et al. 2004 and references therein). In vivo, prp17 deletion results in slow growth at $30^{\circ} \mathrm{C}$ and a temperature sensitive (ts) phenotype at $34^{\circ} \mathrm{C}$; growth defects at both temperatures were suppressed by the cef1V36R allele (Fig. 2G).

Taken together, the suppression of second-step splicing defects due to either multiple intron mutations, lethal alleles of U6 snRNA, or deletion of prp17 argues that the cef1 alleles are general suppressors of second-step defects; indeed, they are among the strongest such suppressors isolated to date.

\section{The cef1 alleles activate selection of alternative $3^{\prime} \mathrm{SS}$}

In addition to improving the efficiency of the second step, primer extension assays of RNA isolated from cells expressing the cef1-V36R allele revealed an additional, slowermigrating mRNA product from the 3'SS gAG/ reporter (Fig. 2B, lane 11), which was identified by RT-PCR and sequencing as an alternatively spliced mRNA. Reduced levels of the same product were also identified in RNA from the cef1-S48R-expressing strain (Fig. 2B, lane 12). This alternatively spliced mRNA utilized a cryptic 3'SS located $15 \mathrm{nt}$ downstream from the branch site and $25 \mathrm{nt}$ upstream of the usual 3'SS position (Fig. 2C). The cryptic 3'SS, CAU/, deviates from the consensus at the last intron position, and such deviation from the consensus typically severely inhibits the second step of splicing. We, therefore, analyzed splicing of the equivalent reporter containing $3^{\prime} \mathrm{SS}$ $\mathrm{UAu} /$ instead of the gAG/ mutation at the original $3^{\prime} \mathrm{SS}$ to compare the effects of cef1 alleles on two competing YAU/ 3'SS (Fig. 2B, lanes 13-15). As expected, the second step for this mutant $3^{\prime}$ SS was strongly inhibited in wildtype cells and was improved by cef1 alleles. As was observed with the 3'SS gAG/ reporter, the upstream cryptic 3'SS, CAU/, was also used in the presence of the cef 1 alleles; in addition, RT-PCR and sequencing of the alternatively spliced mRNA product revealed that two cryptic UUG/ 3'SS also were used. Thus, the cef1 alleles not only improve the second step of splicing for mutated versions of the $3^{\prime} \mathrm{SS}$ at the wild-type position but also enable similar sequences at other positions to be used, an observation strikingly reminiscent of the activation of alternative 3'SS observed in other systems (e.g., Mendes Soares et al. 2006; see Discussion).

\section{The importance of arginine at suppressor positions}

Suppressor mutations in Cef1 might exert their effects through disruption of interactions or by formation of new ones (or both). For example, all tested amino acid substitutions at position R1753 of Prp8 result in suppression of both first-step defects and defects due to prp22 mutations (Schneider et al. 2004; Liu et al. 2007), suggesting that R1753 participates in an interaction that stabilizes the second-step conformation and is disrupted upon mutation. To test such a possibility for the two isolated cef1 suppressor mutations, we randomized V36 and S48 positions and rescreened the pool of mutants for the ability to improve BS-G intron splicing. For position V36, we obtained three colonies, all of which contained V36R; for position S48, each of the 20 colonies selected contained either S48R or $-\mathrm{K}$ (Fig. 4B).

In an independent assay, we introduced a number of point mutations at these two positions and tested their effects on splicing. Consistent with the results of the randomization screen, only V36R and S48R or -K strongly improved the second step (Fig. 4C, lanes 2,14,15), whereas other charged residues, such as aspartic acid or glutamic acid (lanes $7,9,18,19$ ), weakly improved the second step. In all cases, increased levels of spliced mRNA signal detected by primer extension correlated with improved growth in the presence of copper. Together, both the randomization rescreen and direct mutational analyses indicate that only V36R and S48R or -K exert strong suppressor effects. These results are inconsistent with the possibility that V36 and S48 (like Prp8-R1753) participate in stabilizing interactions that are disrupted by any other amino acid at these positions; rather, they suggest that V36R and S48R or -K may form new, stabilizing interactions during the second step that give rise to the observed suppression.

\section{The importance of other positions in the myb domains}

To test whether mutations at other positions in the myb domains might also yield suppressor phenotypes, we used doped DNA oligonucleotides that covered the two aminoterminal myb-like domains to create pools of mutants at near-saturation (Fig. 5A). These pools were rescreened for improved BS-G splicing (Fig. 5B). Again, the strongest suppressor mutations isolated were V36R or S48R (Fig. 5C). One weaker mutant, A37P, adjacent to the V36 position, 


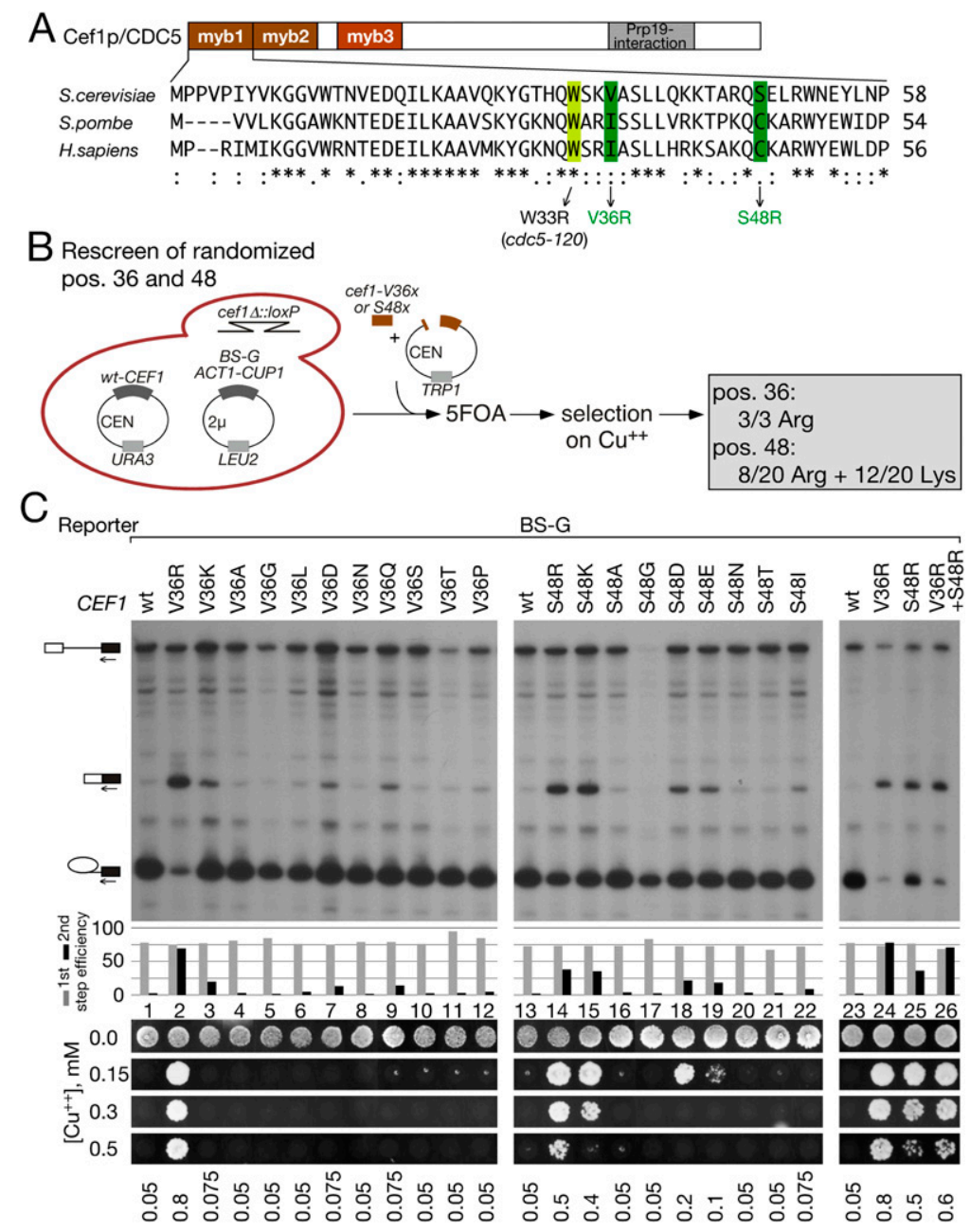

FIGURE 4. Saturation mutagenesis of Cef1 positions 36 and 48 confirms the importance of arginine at these sites. (A) Schematic of domain structure of Cef1/CDC5. Bottom, expansion of the first myb-like domain, alignment of sequences from S. cerevisiae, S. pombe, and Homo sapiens, and indication of positions of mutants identified here (V36R and S48R) and in the cdc5 S. pombe mutant (W33R) (Ohi et al. 1994). $\left(^{*}\right)$ Positions of amino acid identity in all three species, (:) positions of nonidentity but similar amino acid properties. (B) Schematic of randomization of Cef1 positions 36 and 48 by in vivo gap repair, and selection for improvement of BS-G splicing (limiting for the second step of splicing) by growth in the presence of copper. Selection at position 36 yielded only arginine, whereas selection at position 48 yielded arginine and lysine. $(C)$ Primer extension analysis of BS-G reporters from strains carrying various cef1-V36x mutations, $-S 48 x$ mutations. Primer extension and copper growth assays are as described in Figure 2B.

was identified nine times. Several of the isolated alleles contained three or more mutations, and these included $\mathrm{H} 31 \mathrm{~N}$ and W33R substitutions. H31N represents the amino acid found at this position in $S$. pombe and humans, and W33R is identical to the original $c d c 5$ mutation in $S$. pombe (Fig. 5A; Ohi et al. 1994). No cef1 alleles containing mutations in the second myb domain were isolated. Thus, the strongest suppressor mutations across the two myb domains are the original isolates at positions 36 and 48 , whereas some weaker mutants in the first myb domain may represent sites amenable to the evolutionary accommodation of poor second-step substrates (see Discussion).

\section{cef1 alleles and prp8 alleles act antagonistically on the same event}

We have previously described two classes of $\operatorname{prp} 8$ alleles-those that suppress second-step defects ("second-step alleles") and, conversely, those that inhibit the second step and suppress first-step defects ("first-step alleles") (Query and Konarska 2004; Liu et al. 2007). In support of the two-state model of the catalytic spliceosome, the combination of first- and second-step mutations of $\operatorname{prp} 8$ within a single gene resulted in cancellation of the suppressor effects, indicating that the two classes of prp8 alleles affect the same event in the first-to-second-step transition. To test whether the cefl alleles affect the same or a different event as the prp 8 alleles, we combined the cef 1 alleles with each class of $\operatorname{prp} 8$ mutants. Consistent with their having opposing effects during the first-to-second-step transition, the combination of the cefl suppressors of second-step defects with prp8 suppressors of first-step defects rescued the ts phenotype of these prp 8 alleles (Fig. 6A, upper panel); in contrast, the combination of $p r p 8$ second-step alleles and cef1 second-step alleles resulted in a cold-sensitive phenotype, with cef1$V 36 R$ exhibiting a stronger effect than -S48R (Fig. 6A, lower panel).

The splicing effects of these combinations of cef1 and prp8 alleles were visualized by primer extension using the BS-C reporter, which is limiting for both steps of splicing. Whereas second-step alleles of both $p r p 8$ and cef1 improved the second step (Fig. 6B, lanes $2,3,4,7)$ and first-step alleles of prp8 improved the first step and inhibited the second (lanes 10,13,16), combinations of first-step alleles of prp 8 with the second-step alleles of cef1 resulted in a nearly wild-type phenotype for the weaker cef1 allele (S48R) or in a phenotype similar to that of the stronger cef1 allele (V36R) alone (lanes 11,12,14,15,17,18)-i.e., in all cases, combination of a first-step prp8 allele with a secondstep cef 1 allele resulted in an efficiency of the first step that was intermediate between that observed with either allele alone and also resulted in an efficiency of the second step that was intermediate between that observed with either allele alone. Such a cancellation of effects is expected if the two mutations exert opposing effects on the same (or 

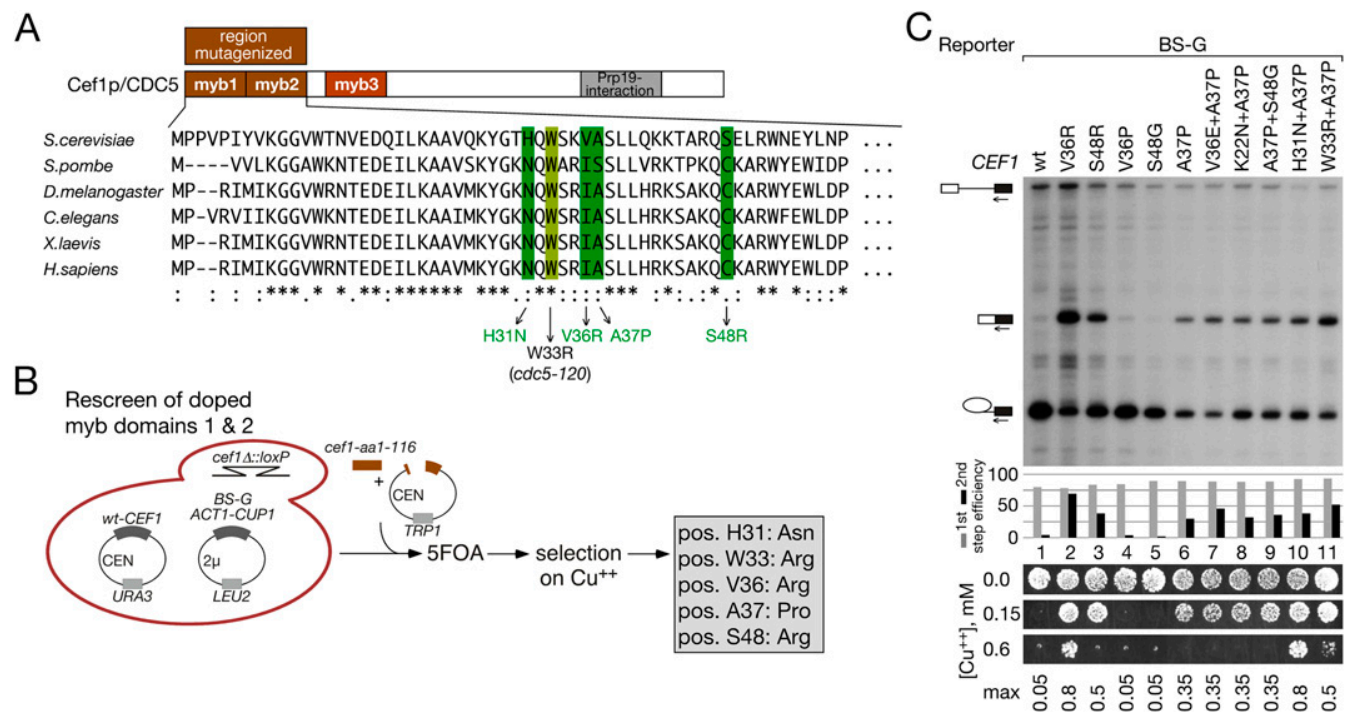

FIGURE 5. Saturation mutagenesis of Cef1 myb-like domains confirms the importance of positions 36 and 48 . (A) Upper, schematic of domain structure of Cef1/CDC5 and the two myb-like domains that were mutagenized. Lower, expansion of the first myb-like domain, alignment of sequences from S. cerevisiae, S. pombe, and H. sapiens, and indication of positions of mutants identified (H31N, W33R, V36R, A37P, and S48R). $\left(^{*}\right)$ Positions of amino acid identity in all three species, (:) positions of nonidentity, but similar amino acid properties. (B) Schematic of randomization of the cef1 myb-like domains 1 and 2 by in vivo gap repair, and selection for improvement of BS-G splicing (limiting for the second step of splicing) by growth in the presence of copper. (C) Primer extension analysis of BS-G reporter from strains carrying various cef1 mutations identified in panel $B$. Primer extension and copper growth assays are as described in Figure 2B. First-step efficiency was calculated as products of the first step/total RNA. Second-step efficiency was calculated as products of the second step/total products from the first step.

tightly coupled) event(s) in the first-to-second-step transition. In addition, the combination of second-step prp8 alleles with second-step cef1 alleles exacerbated the firststep defect of the BS-C reporter (most clearly seen for prp8-161 + cef1-V36R or S48R alleles; Fig. 6B, lanes 4-6). These cancellations of effects described above are in sharp contrast to those observed for combinations of cef 1 alleles with U6 alleles, discussed below.

\section{cef1 alleles act in concert with U6 alleles, affecting a different event}

We tested interactions between cef1 and U6-U57A and -C alleles, which alter the first-to-second-step transition (McPheeters 1996; Query and Konarska 2004; Liu et al. 2007). As discussed above, the cef1 alleles improve the second step for a BS-C intron that is limiting for both steps. U6-U57A generally improves the second but inhibits the first step, whereas U6-U57C strongly improves the first step and inhibits the second (Liu et al. 2007). The combination of U6-U57C and cef1 alleles acted additively to significantly increase mRNA levels, resulting from improvement of both first and second steps-consistent with the strongly improved growth on copper (Fig. 7B, lanes 8-9). A similar additive effect was previously observed for U6-U57C in combination with second-step prp8 alleles, which also improved both first and second steps (Query and Konarska 2004).
Consistent with these results, the combination of U6-U57A (second-step allele) with second-step cef1 alleles resulted in synthetic lethality at $37^{\circ} \mathrm{C}$; in contrast, the combination of U6-U57C (first-step allele) with the second-step cef1 alleles resulted in rescue of the ts phenotype of U6-U57C (Fig. 7A). A similar rescue of the U6-U57C ts phenotype was observed in combination with second-step prp8 alleles (Query and Konarska 2004). Thus, cef1 suppressors exhibit genetic and functional interactions with U6-U57 alleles that are highly analogous to such interactions between U6-U57 and prp8 alleles (Liu et al. 2007): this suggests that the cef1 and U6 alleles affect different events in the first-to-second-step transition, with cef1 alleles affecting the same event(s) as do known prp8 alleles.

\section{DISCUSSION}

Alleles of the NTC component Cef1/CDC5 represent the strongest modulators of spliceosome function identified to date. The two catalytic events of splicing are mediated by two conformations of the spliceosome, separated by rearrangements during which the substrate is repositioned. Genetic and functional interactions between spliceosomal components suggest that cef1 alleles, as well as previously described prp 8 alleles that improve the first or second steps, stabilize or destabilize competing spliceosomal conformations during the catalytic phase of splicing. Modulation of the relative 
A

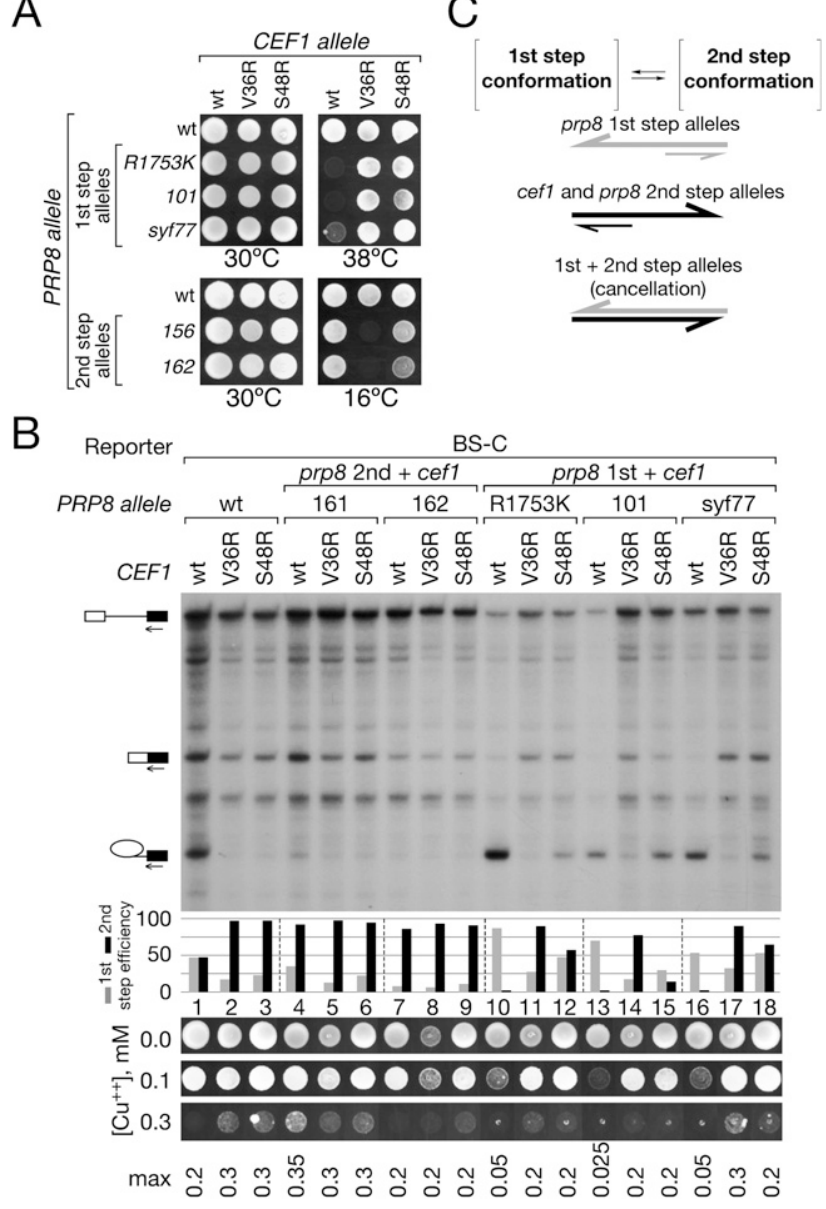

FIGURE 6. The combination of first-step alleles of $\operatorname{prp} 8$ and secondstep alleles of cef1 restores a nearly wild-type pattern of splicing. $(A)$ Genetic interactions between cefl and prp8 alleles. Second-step cef 1 alleles suppress the temperature-sensitive growth defect of first-step prp8 alleles at $38^{\circ} \mathrm{C}$ (upper), whereas cefl alleles are cold-sensitive in combination with second-step prp 8 alleles (lower, $16^{\circ} \mathrm{C}$ ). The temperature-sensitive phenotype of first-step prp 8 alleles in the presence of wild-type CEF1 is suppressed by both of the second-step cef1 alleles (upper). Strains carrying plasmid-borne cef1 and prp 8 alleles were spotted on plates incubated at $16,25,30,37$, and $38^{\circ} \mathrm{C}$. (B) Primer extension analysis and copper growth phenotypes of BS-C reporter (limiting for both steps of splicing) from strains carrying combinations of prp8 first-step alleles (prp8-R1753K, 8-101, and 8-syf77) or second-step alleles (prp8-161 and 8-162) with cef1 second-step alleles (cef1-V36R and -S48R), as indicated. First-step efficiency was calculated as products of the first step/total RNA. Second-step efficiency was calculated as products of the second step/total products from the first step. $(C)$ Schematic of cancellation of altered splicing upon combination of prp8 first-step alleles with cefl second-step alleles.

stabilities of these competing conformations alters splicesite selection and splicing fidelity.

\section{cef1 alleles modulate the catalytic phase of splicing}

Our previously proposed two-state model was based on the identification of two classes of spliceosomal mutations that alter splicing fidelity. One class, second-step alleles, increases the efficiency of the second step of splicing at the expense of the first step. Their functional counterparts, first-
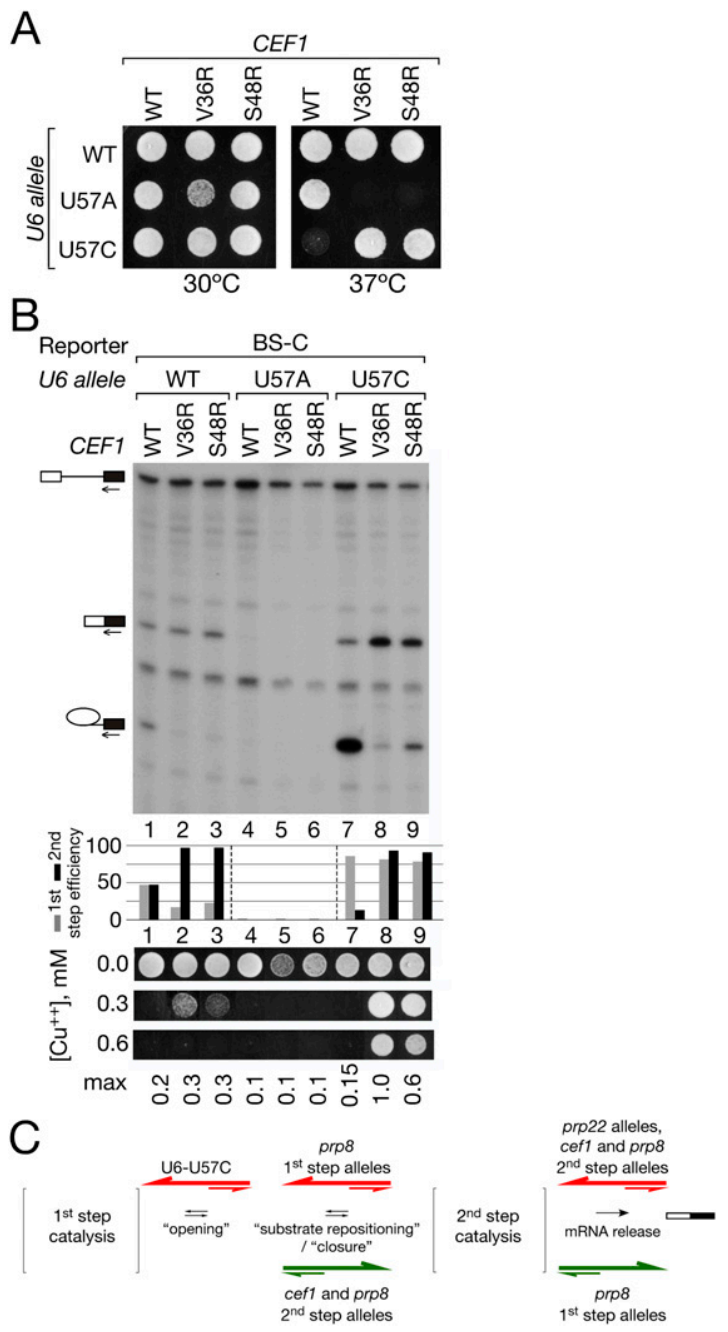

FIGURE 7. cef1 and U6-U57C alleles affect splicing at distinct steps. (A) Genetic interactions between cef1 and U6 snRNA alleles. Secondstep cef1 alleles are synthetically lethal with the second-step U6-U57A allele at $37^{\circ} \mathrm{C}$, whereas the same cef 1 alleles suppress the temperaturesensitive growth defect of the first-step U6-U57C allele at $37^{\circ} \mathrm{C}($ right $)$. $(B)$ Effects of combination of cef1 second-step allele with U6-U57C or U6-U57A on splicing of BS-C reporters. For BS-C introns (limiting for both steps), combinations of U6-U57C and cef1 second-step alleles improve overall splicing (lanes 8-9), in contrast to the combination of prp8 first-step alleles and cef1 second-step alleles (see Fig. 6). First-step efficiency was calculated as products of the first step/total RNA. Second-step efficiency was calculated as products of the second step/ total products from the first step. $(C)$ Proposed contributions of Cef1, Prp8, U6 snRNA, and Prp22 to distinct steps in spliceosomal transitions. cef1 alleles modulate an event in the first-to-second-step transition indistinguishable from that modulated by prp8 alleles; this event is distinct from that modulated by alleles of prp16 and U6 snRNA. We propose that these two distinct events represent the theoretically required opening of the catalytic center and repositioning of first-step products and second-step substrates. The same cef1, $\operatorname{prp} 8$, and U6 alleles, as well as alleles of prp22, alter the exit of mRNA from the second-step spliceosome, which may involve analogous events. 
step alleles, increase the efficiency of the first step at the expense of the second (Query and Konarska 2004; Liu et al. 2007). The existence of two opposing classes of suppressor alleles, each capable of suppressing a wide range of intron mutations, suggests that suppression does not necessarily occur via direct contact between mutated bases/amino acids but rather that the spliceosomal conformations that mediate the first and second steps are in competition with one another. An increased dwell time in one catalytic conformation relative to the other would improve the use of substrates that are suboptimal for the step that is stabilized.

The two-state model predicted that suppressors of second-step defects would exacerbate defects in the subsequent conformational transition, which is facilitated by the spliceosomal ATPase Prp22. Experimental confirmation of this relationship, in turn, provided a means to identify the strongest suppressors as alleles of CEF1. cef1 mutations selected to suppress the second-step defect of a BS-G intron increase the splicing efficiency of all tested intron mutations that impair the second step, and these suppressors inhibit the first step of splicing to a degree commensurate with their second-step stimulation. cef1 alleles also suppressed a variety of known second-step defects that were not due to intron mutation: rescue of lethal (or conditionally lethal) U6 snRNA mutations (A51C, G52C, A59C); complementation of second-step inhibition by prp8 firststep alleles; and rescue of conditional growth defects due to deletion of the second-step protein factor Prp17. These effects are consistent with cef1-V36R and $-S 48 R$ alleles providing an overall relative stabilization to the secondstep conformation (see below). As a corollary, second-step factors like Prp17 can equally be assigned a role in stabilization of the second-step conformation, which might be achieved by a variety of mechanisms. The correlation between the requirement of some of these factors for efficient splicing of introns with an increased branch-to3'SS distance (Brys and Schwer 1996; Schwer and Gross 1998; Sapra et al. 2004) likely reflects a reduced stability of the second-step spliceosome for these introns, due either to weaker $3^{\prime}$ SS binding or to steric impairment caused by a longer branch-to-3'SS RNA strand.

\section{Contribution to molecular events at transitions within the catalytic phase of splicing}

Cef1 is a stable component of the NTC (Tsai et al. 1999; Ohi and Gould 2002; Makarova et al. 2004), a large multicomponent protein complex thought to join the forming spliceosome prior to activation (i.e., formation of U2-U6 base-pairing) and to stabilize the association of U5 and U6 snRNPs with the spliceosome (Chan et al. 2003). It is required prior to step 1 and is part of a minimal salt-stable RNP core that is active for catalysis (Ajuh et al. 2000; Chan et al. 2003; Makarova et al. 2004; Bessonov et al. 2008). In addition to the cef1 alleles described here, deletion of the NTC component Isyl improves the second step and rescues cold-sensitive growth defects due to the prp16-302 allele; because Prp16 facilitates exit from the first-step conformation, this suggests that Isyl stabilizes the first-step conformation (Villa and Guthrie 2005). Mutant alleles of other NTC components were identified as synthetically lethal with deletion of the second-step factor Prp17 (aka CDC40) (Ben-Yehuda et al. 2000), and the corresponding wild-type proteins would, therefore, be predicted to stabilize the second-step conformation. Thus, NTC components must make contributions to stabilization of both first- and second-step spliceosomal conformations.

Our previously proposed model of competition between spliceosomal states suggests that stabilization of one conformation relative to a competing conformation results in improved splicing for substrates limiting for the step for which the spliceosome is stabilized. Stabilization of the second-step conformation relative to the first would result in improved second-step catalysis and a less-efficient first step; likewise, stabilization of the first-step conformation relative to the second would result in improved first-step catalysis and a less-efficient second step. Such relative stabilization of one conformation relative to another could result either from mutations that create new stabilizing interactions or from mutations that disrupt interactions. As mutations are generally more likely to disrupt an interaction than to form a new one, it would seem likely that changes in relative stabilization of spliceosomal conformations are most often due to loss of an interaction that stabilizes a competing conformation. For example, mutation of Prp8 position 1753 from arginine to each of the amino acids tested results in a strong first-step allele (suppressing both first-step defects and defects arising from prp22 mutations [Schneider et al. 2004; Liu et al. 2007]), suggesting that the arginine at position 1753 contributes positively to stabilization of the second-step conformation. However, in the case of the cef 1 mutants described here, both the randomization rescreen and direct mutational analyses indicate that only V36R and S48R or $-\mathrm{K}$ exert strong suppressor effects. These results do not support a model in which V36 and S48 participate in stabilizing interactions that are disrupted by any other amino acid at these positions. Although it is conceivable that arginine and lysine mutations are the only amino acids (and amino acids 36 and 48 the only positions) that could lead to destabilization of the first-step conformation, we consider this a highly unlikely scenario; rather, we favor the model in which positively charged residues at position V36 and S48 may form new, stabilizing interactions during the second step that result in the suppressor effects.

Thus, we favor a model wherein the cef1 alleles described here stabilize the second-step spliceosome, permitting stable binding and catalysis of suboptimal second-step substrates. cef1-V36R stimulates utilization of cryptic 3'SS more than does cef1-S48R; however, the combined results of our assays 
indicate that these two alleles behave similarly, but that cef1$V 36 R$ exerts stronger effects than does cef1-S48R. cef1-V36R is observed to rescue second-step intron defects more effectively than $c e f 1-S 48 R$ by direct visualization of cellular RNA by primer extension analysis and by copper growth reporter assay (with the exception of 3'SS mutations, where cef1-V36R more strongly activates near-cognate cryptic sites, which are out of frame and thus reduce the level of functional mRNA and growth on copper). cef1-V36R also more strongly rescued growth defects due to the U6-A51C and -A59C alleles, which are primarily defective for the second step (Fabrizio and Abelson 1990); and cef1-V36R, but not cef1-S48R, rescued growth defects conferred by deletion of the second-step factor Prp17. In each of these situations, we conclude that the cefl alleles identified here provide a stabilizing interaction to the second-step spliceosome, which otherwise binds less stably to 3'SS mutants, or whose stability is impaired due to U6 mutations or the absence of a second-step factor. The increased stability of a cefl-V36R-containing (and to a lesser extent cef1-S48R-containing) second-step spliceosome would sufficiently extend the dwell time, even on near-cognate sites, either for the second-step spliceosome to become stably engaged or for the catalytic event itself.

We also observed two kinds of properties conferred on splicing of suboptimal substrates upon combination of mutant alleles of cef1, prp8, and U6 snRNA: cancellation and additivity. Cancellation can be explained by combination of mutants that affect the same state oppositely. Combination of first-step prp 8 and second-step cef1 alleles, which independently conferred splicing defects, restored a splicing phenotype similar to that of wild-type cells, whereas combination of prps and cef1 second-step alleles exacerbated their individual effects. Such cancellation of effects suggests altered stability of the same (or a tightly coupled) conformation (Fig. 6C). First-step prp8 alleles could act by destabilization, and second-step prp8 and cef1 alleles could act by stabilization of the second-step conformation; they would also improve and inhibit, respectively, the first step by alteration of competition between the two conformations.

Additivity can be explained by combination of two separate mutations, each of which stabilizes a different catalytic state. In this case, the first step is directly improved, resulting in increased product of the first step (substrate for the second step), which itself may destabilize the first-step conformation and enhance transition to the second-step conformation. Using combinations of cef1 alleles with U6-U57C alleles, both first and second steps were improved, which contrasts dramatically with the results observed above for prp8 and cef1 alleles. Thus, cef1 suppressors exhibit genetic and functional interactions with U6 alleles that are highly analogous to such interactions previously characterized between U6 and prp8 alleles (Liu et al. 2007); taken together, the above data suggest that the cef 1 and prp8 alleles affect the same state of the spliceosome and that cef1 and U6-U57C alleles affect different spliceosomal states (Fig. 7C).

An intermediate in transition between first and second steps is both theoretically required (Liu et al. 2007) and experimentally supported (Hilliker et al. 2007; Perriman and Ares 2007; Mefford and Staley 2009). During the firstto-second-step transition, it is likely that the formation of first-step products (the lariat intermediate and cleaved 5' exon) contributes to the transition and that an altered, or "open," form of the first-step catalytic center disrupts interactions between U6 snRNA and the branch structure (Konarska et al. 2006; Liu et al. 2007). This would be followed by repositioning of the lariat intermediate relative to U6, binding of the 3'SS for the second step, and subsequent "closure" of the catalytic center. A similar series of events would take place again after the second step, with opening of the catalytic center followed by repositioning of products (resulting in mRNA release).

We cannot determine whether the cef1 alleles that affect the second step directly influence the stability of the second catalytic state per se or of this intermediate in transition to the second-step catalytic conformation. That secondstep alleles of cef 1 and prp8 exacerbate prp22 alleles suggests that they stabilize the second-step catalytic conformation (or stabilize what Prp22 acts on); however, we cannot rule out that stabilization of a tightly coupled adjacent state could have similar properties.

\section{The importance of suppressor mutation positions in the Cef1 myb domains}

Saturation mutagenesis of the two amino-terminal myblike domains of Cef1 suggests that V36R and S48R or -K are the only positions and substitutions that yield strong suppressor phenotypes. As discussed above, this suggests that positively charged residues at position V36 and S48 may form new, stabilizing interactions during the second step that result in the suppressor effects. Because mutations in Cef1, like Prp8, generally yield the strongest suppressors of splicing defects, they both may contribute directly to a critical feature of the transition between the two steps of splicing. It is even possible that the introduction of an additional arginine residue into the myb-like domain of Cef1 contributes to stabilization of otherwise unstable RNA-RNA interactions, analogous to the stabilization of RNA-RNA interactions by SR proteins described by Shen and Green (2004, 2007). Recent structural analyses of a Prp8 domain reveal the likely proximity of previously described prp8 mutations to the catalytic center (Pena et al. 2008; Ritchie et al. 2008; Yang et al. 2008); the potent effects of cef1 alleles on splicing suggests that the Cef1 myb-like domain is placed in similar proximity to the core.

A different class of mutations in Cef1 that result in an inhibition of splicing has been previously described; the 
best characterized of these, W52G+W84G, was shown to significantly inhibit splicing of a large number of yeast introns (Burns et al. 2002). The tryptophan residues in myb domains are critical for formation of intra-domain contacts, and the double-tryptophan mutant likely results in loss-of-function (Ohi et al. 1998). In contrast, mutants selected from our screen belong to a different, positionally specific class, and the affected residues, V36 and S48, are likely to contribute less to overall structure of the myblike domain and more to its contacts with other spliceosomal component(s). We anticipate that mutations in additional Cef1 positions and in other spliceosomal factors would belong to the latter class but confer weak phenotypes (e.g., two other suppressors from our screen, which are not alleles of $\operatorname{prp} 8$, cefl, isy1, or any snRNA). For example, we examined the Cef1 residue corresponding to the original S. pombe cef1/cdc5 mutation, W33R, which is thought to affect the cell cycle by altering splicing (Burns et al. 2002), but detected only a mildly improved second step (data not shown). We also identified the W33R and H31N mutations in combination with A37P from our saturation mutagenesis of the two myb-like domains, selecting for an improved second step (Fig. 5); in both cases, the combination resulted in greater secondstep improvement than with $\mathrm{A} 37 \mathrm{P}$ alone, suggesting that all three mutations can weakly improve the second step but that the context of neighboring amino acids may also play a role. $\mathrm{H} 31 \mathrm{~N}$ is the naturally occurring amino acid at position $\mathrm{N} 27$ in $S$. pombe Cef1/Cdc5, suggesting that the wild-type $S$. pombe Cef1/Cdc5 enhances the second step relative to $S$. cerevisiae Cef1. (The mechanism by which these weak alleles alter the second step of splicing need not be the same as that of the strong cefl-V36R and -S48R alleles, discussed above.)

An improved second step of splicing can result in use of suboptimal 3'SS, one manifestation of which is alternative splicing. The phenotype of cefl-V36R closely resembles that of some alternative splicing in higher eukaryotes in that different 3'SS sequences can be used for the second step, after a single branch site is used for the first step (Mendes Soares et al. 2006). Such selection of alternative splice sites in yeast can be achieved by various alterations in the pre-mRNA, the spliceosome, or transcription elongation rate (Umen and Guthrie 1996; Howe et al. 2003; Crotti and Horowitz 2009). Modulation of alternative splicing in metazoan systems might be achieved by similar adjustments to the spliceosome, consistent with the observed changes in alternative splicing in Drosophila and in HeLa cells upon knock-down of levels of basal spliceosome components (Park et al. 2004; Saltzman et al. 2011). One possibility for such alteration of spliceosome function during evolution is that organisms that utilize more diverse splice sites may harbor changes in spliceosome factors similar to those found in the mutant alleles of yeast spliceosomal factors identified in our screens. Although changes corresponding to the strongest cef1 alleles (arginine mutants) are not found in higher organisms, changes corresponding to the weaker alleles are found (e.g., changes in positions H31 and A37, and conservative changes in positions V36 and S48), suggesting that evolutionary changes in spliceosome function have selected for alterations that modulate spliceosome fidelity.

\section{MATERIALS AND METHODS}

\section{Strains and reporter plasmids}

S. cerevisiae strains were derived from the following:

$y$ MK02 [MATa ade2 cup1 $\Delta::$ ura3 his3 leu2 lys2 trp1 ura3 prp22 $\Delta:: l o x P$, p360-22 (PRP22 URA3 CEN ARS)];

$y M K 04$ [MATa ade2 cup1 $\Delta::$ ura3 his3 leu2 lys2 trp1 ura3 cef1M2(V36R) prp22 $\Delta:: 10 x P$, p360-22 (PRP22 URA3 CEN ARS)]; yJU75 (Umen and Guthrie 1996) [MATa, ade2 cup1A::ura3 his3 leu2 lys2 prp84::LYS2 trp1, pMK8-1 (PRP8 HIS3 CEN ARS)];

$y C Q 05$ [MATa, ade2 cup1 $\Delta::$ ura3 his3 leu2 lys2 prp8 $\Delta::$ LYS2 trp1 ura3 U6 $\Delta:$ KAN, pCC130 (U6 URA3 CEN ARS), pMK8-1 (PRP8 HIS3 CEN ARS)];

$y C Q 11$ [MATa, ade2 cup1 $\Delta::$ ura3 his3 leu2 lys2 prp8 $\Delta::$ LYS2 trp1 ura3 U6 $\Delta:: K A N$, cef1-V36R, pCC130 (U6 URA3 CEN ARS), pMK8-1 (PRP8 HIS3 CEN ARS)];

$y C Q 12$ [MATa, ade2 cup1 $\Delta::$ ura3 his3 leu2 lys2 prp8 $\Delta::$ LYS2 trp1 ura3 U6 $\Delta:: K A N$, cef1-S48R, pCC130 (U6 URA3 CEN ARS), pMK8-1 (PRP8 HIS3 CEN ARS)];

yCQ16 [MATa ade2 cup1 $::$ ura3 his3 leu2 lys2 trp1 ura3 cef1 $\Delta::$ loxP, pRS316-CEF1 (CEF1 URA3 CEN ARS)];

containing plasmid-borne alleles of cef1, prp22, prp8, and U6 (snr6) as indicated in the figures. ACT1-CUP1 reporter plasmids (Lesser and Guthrie 1993) with 5'SS, BS, or 3'SS mutations were as described (Query and Konarska 2004).

\section{Copper assays}

Cultures were grown to midlog phase in -Leu medium, diluted to $0.2 \mathrm{OD}$, and equal volumes were dropped onto - Leu plates containing $\mathrm{CuSO}_{4}$ ranging from 0 to $2.0 \mathrm{mM}$ (Lesser and Guthrie 1993). Plates were scored and photographed after $3 \mathrm{~d}$ at $30^{\circ} \mathrm{C}$.

\section{RNA analysis}

Primer extensions were carried out as described (Query and Konarska 2004) using primer YAC6 5'-GGCACTCATGAC CTTC-3', complementary to exon 2 of ACT1. Extension products were separated in $7 \%$ polyacrylamide/ $8 \mathrm{M}$ urea gels and visualized by autoradiography. To identify the cryptic $3^{\prime} \mathrm{SS}$ utilized in the presence of the cefl-V36R allele, the alternatively spliced mRNA band was eluted from the primer extension gel, PCR-amplified with $5^{\prime}$ and $3^{\prime}$ exon-specific primers, and sequenced. Firststep efficiency was calculated as products of the first-step (lariat intermediates+mRNA)/total RNA (lariat intermediates+mRNA+ pre-mRNA). Second-step efficiency was calculated as products of the second step (mRNA)/total products from the first step (lariat intermediates+mRNA). 


\section{ACKNOWLEDGMENTS}

We are grateful to Beate Schwer for prp22 mutant plasmids, Greg Prelich for the wild-type genomic library, and Duncan J. Smith, Alberto Moldón Vara, and Beth Moorefield for helpful discussions and critical readings of the manuscript. This work was supported by NIH grant GM57829 to C.C.Q., by NIH grant GM49044 to M.M.K., and by a Cancer Center Support (core) grant from the NCI to AECOM. C.C.Q. is a scholar of the Irma T. Hirschl Trust.

Received July 19, 2011; accepted January 25, 2012.

\section{REFERENCES}

Ajuh P, Kuster B, Panov K, Zomerdijk JC, Mann M, Lamond AI 2000. Functional analysis of the human CDC5L complex and identification of its components by mass spectrometry. EMBO J 19: 6569-6581.

Ben-Yehuda S, Russell CS, Dix I, Beggs JD, Kupiec M. 2000. Extensive genetic interactions between PRP8 and PRP17/CDC40, two yeast genes involved in pre-mRNA splicing and cell cycle progression. Genetics 154: 61-71.

Bessonov S, Anokhina M, Will CL, Urlaub H, Lührmann R. 2008. Isolation of an active step I spliceosome and composition of its RNP core. Nature 452: 846-850.

Brys A, Schwer B. 1996. Requirement for SLU7 in yeast pre-mRNA splicing is dictated by the distance between the branchpoint and the 3' splice site. RNA 2: 707-717.

Burns CG, Ohi R, Mehta S, O’Toole ET, Winey M, Clark TA, Sugnet CW, Ares M Jr, Gould KL. 2002. Removal of a single $\alpha$-tubulin gene intron suppresses cell cycle arrest phenotypes of splicing factor mutations in Saccharomyces cerevisiae. Mol Cell Biol 22: 801-815.

Chan SP, Kao DI, Tsai WY, Cheng SC. 2003. The Prp19p-associated complex in spliceosome activation. Science 302: 279-282.

Collins CA, Guthrie C. 1999. Allele-specific genetic interactions between Prp8 and RNA active site residues suggest a function for Prp8 at the catalytic core of the spliceosome. Genes Dev 13: 1970-1982.

Crotti LB, Horowitz DS. 2009. Exon sequences at the splice junctions affect splicing fidelity and alternative splicing. Proc Natl Acad Sci 106: $18954-18959$.

Fabrizio P, Abelson J. 1990. Two domains of yeast U6 small nuclear RNA required for both steps of nuclear precursor messenger RNA splicing. Science 250: 404-409.

Grainger RJ, Beggs JD. 2005. Prp8 protein: At the heart of the spliceosome. RNA 11: 533-557.

Hilliker AK, Staley JP. 2004. Multiple functions for the invariant AGC triad of U6 snRNA. RNA 10: 921-928.

Hilliker AK, Mefford MA, Staley JP. 2007. U2 toggles iteratively between the stem IIa and stem IIc conformations to promote premRNA splicing. Genes Dev 21: 821-834.

Howe KJ, Kane CM, Ares M Jr. 2003. Perturbation of transcription elongation influences the fidelity of internal exon inclusion in Saccharomyces cerevisiae. RNA 9: 993-1006.

Konarska MM, Query CC. 2005. Insights into the mechanisms of splicing: More lessons from the ribosome. Genes Dev 19: 22552260.

Konarska MM, Vilardell J, Query CC. 2006. Repositioning of the reaction intermediate within the catalytic center of the spliceosome. Mol Cell 21: 543-553.

Koodathingal P, Novak T, Piccirilli JA, Staley JP. 2010. The DEAH box ATPases Prp16 and Prp43 cooperate to proofread $5^{\prime}$ splice site cleavage during pre-mRNA splicing. Mol Cell 39: 385-395.

Lesser CF, Guthrie C. 1993. Mutational analysis of pre-mRNA splicing in Saccharomyces cerevisiae using a sensitive new reporter gene, CUP1. Genetics 133: 851-863.
Liu L, Query CC, Konarska MM. 2007. Opposing classes of prp8 alleles modulate the transition between the catalytic steps of premRNA splicing. Nat Struct Mol Biol 14: 519-526.

Madhani HD, Guthrie C. 1992. A novel base-pairing interaction between $\mathrm{U} 2$ and U6 snRNAs suggests a mechanism for the catalytic activation of the spliceosome. Cell 71: 803-817.

Madhani HD, Bordonne R, Guthrie C. 1990. Multiple roles for U6 snRNA in the splicing pathway. Genes Dev 4: 2264-2277.

Makarova OV, Makarov EM, Urlaub H, Will CL, Gentzel M, Wilm M, Lührmann R. 2004. A subset of human 35S U5 proteins, including Prp19, function prior to catalytic step 1 of splicing. EMBO J 23: 2381-2391.

Mayas RM, Maita H, Staley JP. 2006. Exon ligation is proofread by the DExD/H-box ATPase Prp22p. Nat Struct Mol Biol 13: 482490

McPheeters DS. 1996. Interactions of the yeast U6 RNA with the premRNA branch site. RNA 2: 1110-1123.

Mefford MA, Staley JP. 2009. Evidence that U2/U6 helix I promotes both catalytic steps of pre-mRNA splicing and rearranges in between these steps. RNA 15: 1386-1397.

Mendes Soares LM, Zanier K, Mackereth C, Sattler M, Valcárcel J. 2006. Intron removal requires proofreading of $\mathrm{U} 2 \mathrm{AF} / 3^{\prime}$ splice site recognition by DEK. Science 312: 1961-1965.

Nurse P, Thuriaux P, Nasmyth K. 1976. Genetic control of the cell division cycle in the fission yeast Schizosaccharomyces pombe. Mol Gen Genet 146: 167-178.

Ohi MD, Gould KL. 2002. Characterization of interactions among the Cef1p-Prp19p-associated splicing complex. RNA 8: 798815 .

Ohi R, McCollum D, Hirani B, Den Haese GJ, Zhang X, Burke JD, Turner K, Gould KL. 1994. The Schizosaccharomyces pombe $c d c 5^{+}$ gene encodes an essential protein with homology to c-Myb. EMBO J 13: 471-483.

Ohi R, Feoktistova A, McCann S, Valentine V, Look AT, Lipsick JS, Gould KL. 1998. Myb-related Schizosaccharomyces pombe cdc5p is structurally and functionally conserved in eukaryotes. Mol Cell Biol 18: 4097-4108.

Park JW, Parisky K, Celotto AM, Reenan RA, Graveley BR. 2004. Identification of alternative splicing regulators by RNA interference in Drosophila. Proc Natl Acad Sci 101: 15974-15979.

Pena V, Rozov A, Fabrizio P, Lührmann R, Wahl MC. 2008. Structure and function of an RNase $\mathrm{H}$ domain at the heart of the spliceosome. EMBO J 27: 2929-2940.

Perriman RJ, Ares M Jr. 2007. Rearrangement of competing U2 RNA helices within the spliceosome promotes multiple steps in splicing. Genes Dev 21: 811-820.

Query CC, Konarska MM. 2004. Suppression of multiple substrate mutations by spliceosomal prp8 alleles suggests functional correlations with ribosomal ambiguity mutants. Mol Cell 14: 343354.

Ritchie DB, Schellenberg MJ, Gesner EM, Raithatha SA, Stuart DT, Macmillan AM. 2008. Structural elucidation of a PRP8 core domain from the heart of the spliceosome. Nat Struct Mol Biol 15: 1199-1205.

Saltzman AL, Pan Q, Blencowe BJ. 2011. Regulation of alternative splicing by the core spliceosomal machinery. Genes Dev 25: 373384.

Sapra AK, Arava Y, Khandelia P, Vijayraghavan U. 2004. Genomewide analysis of pre-mRNA splicing: Intron features govern the requirement for the second-step factor, Prp17 in Saccharomyces cerevisiae and Schizosaccharomyces pombe. J Biol Chem 279: 5243752446.

Schneider S, Campodonico E, Schwer B. 2004. Motifs IV and V in the DEAH box splicing factor Prp22 are important for RNA unwinding, and helicase-defective Prp22 mutants are suppressed by Prp8. J Biol Chem 279: 8617-8626.

Schwer B, Gross CH. 1998. Prp22, a DExH-box RNA helicase, plays two distinct roles in yeast pre-mRNA splicing. EMBO J 17: 20862094. 
Schwer B, Guthrie C. 1991. PRP16 is an RNA-dependent ATPase that interacts transiently with the spliceosome. Nature 349: 494499.

Shen H, Green MR. 2004. A pathway of sequential arginine-serine-rich domain-splicing signal interactions during mammalian spliceosome assembly. Mol Cell 16: 363-373.

Shen H, Green MR. 2007. RS domain-splicing signal interactions in splicing of U12-type and U2-type introns. Nat Struct Mol Biol 14: 597-603.

Siatecka M, Reyes JL, Konarska MM. 1999. Functional interactions of Prp8 with both splice sites at the spliceosomal catalytic center. Genes Dev 13: 1983-1993.

Smith DJ, Query CC, Konarska MM. 2008. "Nought may endure but mutability": Spliceosome dynamics and the regulation of splicing. Mol Cell 30: 657-666.
Tsai WY, Chow YT, Chen HR, Huang KT, Hong RI, Jan SP, Kuo NY, Tsao TY, Chen CH, Cheng SC. 1999. Ceflp is a component of the Prp19p-associated complex and essential for pre-mRNA splicing. J Biol Chem 274: 9455-9462.

Umen JG, Guthrie C. 1996. Mutagenesis of the yeast gene PRP8 reveals domains governing the specificity and fidelity of $3^{\prime}$ splice site selection. Genetics 143: 723-739.

Villa T, Guthrie C. 2005. The Isylp component of the NineTeen complex interacts with the ATPase Prp16p to regulate the fidelity of pre-mRNA splicing. Genes Dev 19: 1894-1904.

Wahl MC, Will CL, Lührmann R. 2009. The spliceosome: Design principles of a dynamic RNP machine. Cell 136: 701-718.

Yang K, Zhang L, Xu T, Heroux A, Zhao R. 2008. Crystal structure of the $\beta$-finger domain of Prp8 reveals analogy to ribosomal proteins. Proc Natl Acad Sci 105: 13817-13822. 

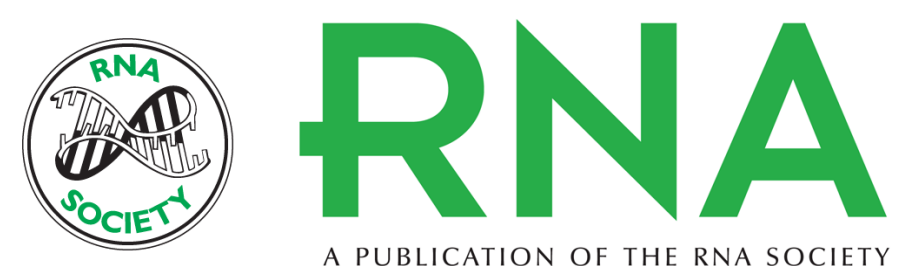

A PUBLICATION OF THE RNA SOCIETY

\section{CEF1/CDC5 alleles modulate transitions between catalytic conformations of the spliceosome}

Charles C. Query and Maria M. Konarska

RNA 2012 18: 1001-1013 originally published online March 8, 2012

Access the most recent version at doi:10.1261/rna.029421.111

\section{References This article cites 48 articles, 28 of which can be accessed free at:} http://rnajournal.cshlp.org/content/18/5/1001.full.html\#ref-list-1

\section{License}
Email Alerting Receive free email alerts when new articles cite this article - sign up in the box at the Service top right corner of the article or click here.

\section{III!" PI Providing Precise Solutions tor your research.}

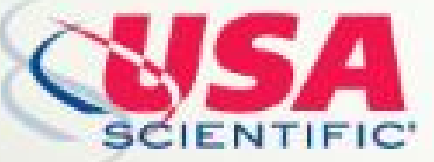

To subscribe to $R N A$ go to:

http://rnajournal.cshlp.org/subscriptions 